\title{
Comparación de la composición química proximal de la carne de Anadara tuberculosa y $A$. similis (Bivalvia: Arcidae) de Chomes, Puntarenas, Costa Rica
}

\section{Comparison of proximate chemical composition of Anadara tuberculosa and $A$. similis (Bivalvia: Arcidae) from Chomes, Puntarenas, Costa Rica}

\author{
Rafael A. Cruz ${ }^{1 *}$, Cristian Fonseca Rodríguez ${ }^{1}$ y Fabián Chavarría-Solera ${ }^{1}$
}

\begin{abstract}
RESUMEN
Se estudiaron la variación estacional de la composición química proximal y el contenido energético de la carne de las pianguas Anadara tuberculosa y A. similis. Mensualmente, entre marzo de 2010 y febrero de 2011, se recolectaron de 15 a 20 ejemplares de cada especie. La composición proximal se determinó siguiendo la metodología recomendada por la Asociación Oficial de Químicos Analíticos (AOAC). El valor promedio de las proteínas de la carne de $A$. similis fue de $57.9 \pm 7.2 \%$, carbohidratos $25.6 \pm 4.4 \%$, cenizas $6.87 \pm 0.23 \%$ y lípidos $9.2 \pm 0.2 \%$. El contenido calórico promedio fue de $5.3 \pm 0.2 \mathrm{kcal} / \mathrm{g}$. Para A. tuberculosa se calculó $61.6 \%$ de proteínas, $21.6 \%$ de carbohidratos, $8.8 \%$ de cenizas, $7.9 \%$ de lípidos; el valor calórico fue de 5.2 $\mathrm{kcal} / \mathrm{g}$. Se puede concluir que tanto A. tuberculosa como A. similis presentan valores nutricionales óptimos para el consumo humano, por su bajo contenido de grasa y su aceptable contenido proteico.
\end{abstract}

Palabras claves: Composición química proximal, valor calórico, Anadara tuberculosa, Anadara similis, Costa Rica.

\begin{abstract}
The seasonal variation in proximate biochemical composition and energy content of Anadara tuberculosa and A. similis were studied between March 2010 and February 2011 on a monthly basis. Fifteen to twenty individuals of each species were collected monthly. The proximate composition was determined following the methodology recommended by the Association of Official Analytical Chemists (AOAC). The average value of meat protein in A. similis was $57.9 \pm 7.2 \%$, carbohydrate $25.6 \pm 4.4 \%$, ash $6.87 \pm 0.23 \%$, and lipids $9.2 \pm 0.2 \%$. The average caloric content was $5.3 \pm 0.2 \mathrm{kcal} / \mathrm{g}$. For $A$. tuberculosa the values were protein $61.6 \%$, carbohydrate $21.6 \%$, ash $8.8 \%$, and lipids $7.9 \%$. The caloric value was $5.2 \mathrm{kcal} / \mathrm{g}$. It can be concluded that both $A$. tuberculosa and $A$. similis have optimal nutritional value for human consumption, due to their low lipid and high protein contents.
\end{abstract}

Keywords: Proximate biochemical composition, caloric content, Anadara tuberculosa, A. similis, Costa Rica.

\footnotetext{
1 Universidad Nacional, Escuela de Ciencias Biológicas, Estación de Biología Marina, Puntarenas, Costa Rica. rcruzsoto47@gmail.com*, cfonseca@una.ac.cr, fbnchs@gmail.com
}

Recibido 23-IX-2011

Aceptado 6-VIII-2012

DOI: http://dx.doi.org/10.15359/revmar.4.6 


\section{INTRODUCCIÓN}

Anadara tuberculosa (Sowerby, 1833) es un molusco bivalvo que habita en los ecosistemas de manglar y posee una amplia distribución en la costa del Pacífico de América, desde Laguna San Ignacio, Baja California Sur, México, hasta Punta Telégrafo, Perú (Skoglund, 2001); representa el molusco bivalvo de mayor importancia comercial a lo largo de su distribución (MacKenzie, 2001). Además, constituye una especie con grandes posibilidades para el desarrollo pesquero (Campos et al. 1990; García-Domínguez et al. 2008).

En Costa Rica, A. tuberculosa, conocida como piangua, es la base de una pesquería exclusivamente artesanal, catalogada como una fuente de empleo para muchas personas, en especial mujeres y niños de estratos socioeconómicos muy bajos, identificados como los más pobres. Además, esta especie es la de mayor consumo en el país y a lo largo de su distribución, siendo el principal plato en forma de ceviche (Fernández, 1983; MacKenzie, 2001).

En el Pacífico de Costa Rica, la especie Anadara similis (C. B. Adams, 1852) se encuentra mezclada con A. tuberculosa y, en general, representa entre un $10-15 \%$ de la pesquería total. Se distribuye desde Boca de Ceuta, Sinaloa, México, hasta Tumbes, Perú (Skoglund, 2001). En Costa Rica se localiza en mayor o menor grado en todos los manglares de la costa del Pacífico. A. similis es una especie que tiene su sangre de color rojizo, la carne es rosada y, además, es más blanda que la de $A$. tuberculosa, lo que redunda en mejores características para el consumo que esta última. A pesar de ser un recurso artesanal de mucha importancia, existe poco conocimiento biológico de esta especie, que sería de gran interés si se piensa en un adecuado manejo.
La mayoría de los trabajos científicos sobre A. tuberculosa en Costa Rica se han centrado en aspectos biométricos, crecimiento y poblacionales (Cruz, 1982; Cruz \& Palacios, 1983; Villalobos \& Báez, 1983; Campos et al. 1990; Vega, 1994; Silva \& Bonilla, 2001), en la biología reproductiva (Cruz, 1984a; Ampie \& Cruz, 1989), sobre contaminación microbiana (Fernández \& Brunker, 1977; Fernández, 1983; Rodríguez \& Antillón, 1989; Wong et al. 1997; Herrero et al. 1999). En lo que se refiere a la composición química solo se localizó el trabajo de Carballo-Avendaño \& Herrera-Ramírez (1996). Sobre $A$. similis solo se han localizado los estudios de Cruz (1984b) sobre aspectos reproductivos y el de Silva \& Bonilla (2001) sobre abundancia y morfometría.

Teniendo en cuenta su importancia pesquera y social es necesario realizar estudios más integrales que incluyan, entre otros, los aspectos relacionados con la composición nutricional, por lo que el objetivo de este trabajo fue comparar la composición nutricional de estas dos especies.

\section{MATERIALES Y MÉTODOS}

Entre agosto de 2009 y julio de 2010 , se recolectaron entre 15 y 20 ejemplares de $A$. tuberculosa y de $A$. similis, de un banco natural en el manglar de Chomes, al este del Golfo de Nicoya, Puntarenas, Costa Rica (10²'30" - $10^{\circ} 4^{\prime} 00^{\prime \prime} \mathrm{N} \mathrm{y}$ $84^{\circ}$ 6' 30" O).

Los ejemplares de ambas especies fueron transportados vivos en una hielera al laboratorio, donde se les determinó la longitud total (máxima medida desde el eje anterior-posterior) utilizando un vernier ( $\pm 0.05 \mathrm{~mm}$ de precisión). Posteriormente, se les determinó el peso total con una balanza $( \pm 0.01 \mathrm{~g}$ de precisión) 
y luego se les removió la carne, la que se drenó por 10 minutos con papel adsorbente para luego pesarla. El tejido de todos los ejemplares fue deshidratado en un horno a $100^{\circ} \mathrm{C}$ por 24 horas para determinar el peso seco, utilizando una balanza analítica $( \pm 0.01 \mathrm{~g})$. El material seco se molió y homogeneizó con un mortero para realizar los análisis bioquímicos por triplicado.

Para la caracterización química proximal de los componentes de la carne, se realizaron determinaciones de proteínas, carbohidratos, cenizas, lípidos y humedad, según la metodología de AOAC (1984). El nitrógeno se determinó por el método de Kjendhal y se convirtió a proteína multiplicando por el factor 6.25 (Crisp, 1971). Los lípidos fueron obtenidos usando un extractor Soxhlet (Lab-Line Instruments, Inc., ILL, USA) con éter de petróleo. Las cenizas se obtuvieron por calcinación lenta, incrementando la temperatura hasta $500^{\circ} \mathrm{C}$, donde se mantuvo por 12 horas (Crisp, 1971). Los carbohidratos fueron calculados por diferencia, luego de calcular el porcentaje de lípidos, proteínas y cenizas. El valor calórico de la carne seca se calculó usando los factores de conversión recomendados para moluscos: $5.7 \mathrm{kcal} / \mathrm{g}$ para proteínas, $4.2 \mathrm{kcal} / \mathrm{g}$ para carbohidratos y $9.5 \mathrm{kcal} / \mathrm{g}$ para lípidos (Lucas \& Beninger, 1985).

El tratamiento estadístico de los datos se realizó utilizando el paquete estadístico STATGRAPHICS ${ }^{\circledR}$ Centurion XV (StatPoint, 2006), mediante el cual se determinaron las correlaciones (Spearman) entre los porcentajes de proteínas, lípidos, cenizas, carbohidratos y el índice de condición (IC) a lo largo del periodo de muestreo. El supuesto de normalidad de los datos fue probado mediante la prueba de ShapiroWilks y la homogeneidad de la varianza fue evaluada usando la prueba de Levene.
Para cada parámetro evaluado se realizó un ANDEVA de una vía, seguida de comparaciones múltiples de Tukey para determinar si existe diferencia significativa entre la composición bioquímica de A. tuberculosa y de $A$. similis durante los meses de muestreos a un nivel de significancia del 5\%. Los datos son reportados como promedios \pm deviación estándar.

\section{RESULTADOS}

A lo largo del periodo de muestreo se analizó un total de 186 especímenes de $A$. tuberculosa y 160 especímenes de $A$. similis. En el cuadro 1 se observan la variación mensual de los valores promedio de la longitud total, el peso total y el peso fresco de la muestra de la población de $A$. tuberculosa y de $A$. similis. La longitud promedio mensual de los ejemplares de $A$. tuberculosa varió entre $44.1 \pm 4.1 \mathrm{~mm}$ y $51.9 \pm$ $6.0 \mathrm{~mm}$ con un promedio de $46.3 \pm 3 \mathrm{~mm}$, el peso total promedio fue de $24.3 \pm 5.9 \mathrm{~g}$, variando entre $17.7 \pm 7.4 \mathrm{~g}$ y $35.2 \pm 16.7 \mathrm{~g}$, el peso fresco varió entre $5.4 \pm 1.6 \mathrm{~g}$ y 9.1 $\pm 3.9 \mathrm{~g}$ para un promedio de $7.3 \pm 1.3 \mathrm{~g}$. Para A. similis, la longitud de los ejemplares varió entre $43.4 \pm 0.8 \mathrm{~mm}$ y $52.0 \pm 2.6$ $\mathrm{mm}$ con un promedio de $47.9 \pm 2.5 \mathrm{~mm}$, el peso total promedio fue de $22.5 \pm 5.0 \mathrm{~g}$, variando entre $15.0 \pm 4.9 \mathrm{~g}$ y $31.9 \pm 7.4 \mathrm{~g}$, el peso fresco varió entre $4.0 \pm 1.3 \mathrm{~g}$ y 8.3 $\pm 2.0 \mathrm{~g}$ para un promedio de $6.3 \pm 1.2 \mathrm{~g}$. No se encontraron diferencias estadísticamente significativas entre las medias de la longitud total, el peso total y el peso fresco de ambas especies $(P>0.05)$.

En la figura 1 se pueden ver las variaciones mensuales de los componentes bioquímicos proximales de $A$. tuberculosa y de $A$. similis. Ambas especies presentaron el máximo porcentaje de humedad en noviembre $(88.7 \pm 2.55 \%$ y $82.2 \pm 2.74 \%)$ 

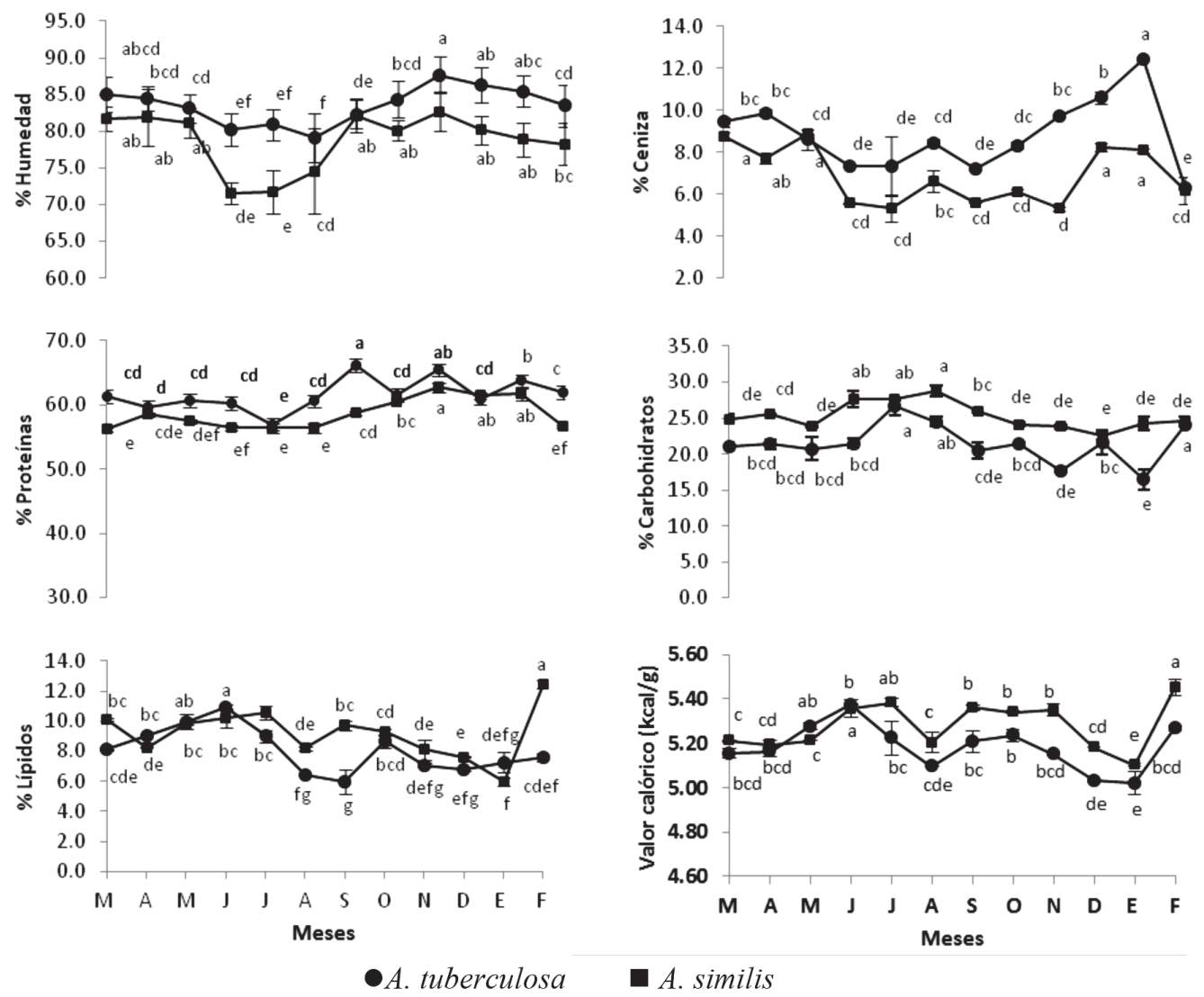

Fig. 1. Variación mensual de los componentes bioquímicos proximales de la carne de Anadara tuberculosa y de $A$. similis. Datos son expresados como promedio \pm D.E. de tres repeticiones. ${ }^{\text {a-g }}$ Diferentes letras en una misma serie de datos para cada especie indican diferencia significativa $(P<0.05)$

Fig. 1. Monthly change in proximate biochemical components of Anadara tuberculosa and A. similis. Data is expressed as mean \pm SD three replications. ${ }^{\mathrm{a}-\mathrm{g}}$ Different letters in the same set of data for each species indicate significant difference $(P<0.05)$

para decrecer significativamente (Tukey, $P<0.05)$ hasta agosto $(79.2 \%)$ en $A$. tuberculosa y hasta junio (71.6\%) en $A$. similis. Después de la humedad, el principal constituyente de la carne en ambas especies fueron las proteínas, A. tuberculosa exhibe el máximo porcentaje en setiembre $(66.2 \pm 0.42 \%)$, mientras que en $A$. similis el máximo porcentaje lo presenta en noviembre $(62.8 \pm 0.78 \%)$. Estos máximos porcentajes mostraron diferencias significativas con respecto a los valores mínimos de ambas especies (Tukey, $P<0.05$ ).
El contenido de lípidos fluctuó entre 10.9 $\pm \%$ y $6.0 \pm \%$ en $A$. tuberculosa y entre $12.45 \pm \%$ y $6.0 \pm \%$ en $A$. similis, variando a través del año (Tukey, $P<0.05$ ) (Fig. 1). A. tuberculosa presentó porcentajes significativamente altos (Tukey, $P<0.05$ ) de carbohidratos en junio, mientras que $A$. similis lo hace en agosto, precisamente en los mismos meses en que las proteínas exhiben los mínimos valores, encontrándose para las dos especie una correlación negativa entre estos componentes $(\mathrm{r}=-0.7, P<$ $0.05)$. El contenido de ceniza de la carne 
Cuadro 1. Variación mensual de longitud total, peso total y peso fresco de $A$. tuberculosa $\mathrm{y} A$. similis (promedio \pm desv. est., $\mathrm{n}=15$ ). $\mathrm{LT}=$ longitud, $\mathrm{PT}=$ peso total, $\mathrm{PF}=$ peso fresco de la carne

Table 1 . Monthly variation in total length, total weight and fresh weight of $A$. tuberculosa and $A$. similis (mean \pm s.d., $\mathrm{n}=15$ ). $\mathrm{SL}=$ shell length, $\mathrm{TW}=$ total weight, $\mathrm{FW}=$ fresh weight

\begin{tabular}{|c|c|c|c|c|c|c|}
\hline \multirow[b]{2}{*}{ Meses } & \multicolumn{3}{|c|}{ A. tuberculosa } & \multicolumn{3}{|c|}{ A. similis } \\
\hline & LT(mm) & PT(g) & $P F(g)$ & LT(mm) & PT(g) & $\mathrm{PF}(\mathrm{g})$ \\
\hline marzo & $44.4 \pm 6.1$ & $23.6 \pm 10.9$ & $7.0 \pm 3.0$ & $46.0 \pm 3.9$ & $18.3 \pm 6.3$ & $7.2 \pm 2.1$ \\
\hline abril & $45.6 \pm 4.2$ & $21.6 \pm 6.2$ & $6.6 \pm 1.7$ & $43.4 \pm 0.8$ & $17.1 \pm 2.5$ & $6.7 \pm 1.2$ \\
\hline mayo & $44.1 \pm 4.1$ & $17.7 \pm 7.4$ & $5.4 \pm 1.6$ & $48.0 \pm 2.9$ & $19.0 \pm 5.1$ & $6.7 \pm 1.6$ \\
\hline junio & $48.9 \pm 6.9$ & $27.7 \pm 13.7$ & $8.4 \pm 3.7$ & $48.3 \pm 2.0$ & $23.1 \pm 5.0$ & $5.8 \pm 1.4$ \\
\hline julio & $51.3 \pm 5.6$ & $35.2 \pm 16.7$ & $9.1 \pm 3.9$ & $47.2 \pm 3.2$ & $15.0 \pm 4.9$ & $4.0 \pm 1.3$ \\
\hline agosto & $51.9 \pm 6.0$ & $31.6 \pm 11.9$ & $8.4 \pm 4.0$ & $47.2 \pm 3.8$ & $20.6 \pm 5.9$ & $4.8 \pm 1.3$ \\
\hline setiembre & $45.2 \pm 5.3$ & $23.7 \pm 10.0$ & $6.4 \pm 2.4$ & $49.0 \pm 3.1$ & $24.1 \pm 5.3$ & $6.5 \pm 1.4$ \\
\hline octubre & $47.3 \pm 6.6$ & $28.0 \pm 13.7$ & $8.5 \pm 4.0$ & $50.4 \pm 3.2$ & $27.5 \pm 6.7$ & $6.5 \pm 1.6$ \\
\hline noviembre & $47.1 \pm 6.2$ & $27.1 \pm 11.4$ & $8.8 \pm 3.5$ & $48.5 \pm 3.9$ & $24.9 \pm 7.6$ & $7.5 \pm 3.0$ \\
\hline diciembre & $43.7 \pm 4.1$ & $20.9 \pm 6.3$ & $6.2 \pm 1.5$ & $47.8 \pm 3.5$ & $20.5 \pm 4.9$ & $5.5 \pm 1.6$ \\
\hline enero & $43.9 \pm 5.3$ & $19.5 \pm 8.3$ & $6.3 \pm 2.1$ & $50.5 \pm 4.4$ & $28.1 \pm 6.9$ & $6.0 \pm 1.9$ \\
\hline febrero & $44.2 \pm 6.1$ & $20.5 \pm 12.7$ & $6.2 \pm 3.4$ & $52.0 \pm 2.6$ & $31.9 \pm 7.4$ & $8.3 \pm 2.0$ \\
\hline Promedio & $46.3 \pm 3.2$ & $24.3 \pm 5.9$ & $7.3 \pm 1.3$ & $47.9 \pm 2.5$ & $22.5 \pm 5.0$ & $6.3 \pm 1.2$ \\
\hline
\end{tabular}

de $A$. tuberculosa y $A$. similis mostró un decrecimiento significativo (Tukey, $P<$ $0.05)$ en febrero-julio (6.3 - 5.3\%) y un incremento significativo (Tukey, $P<0.05$ ) en enero-mayo (12.5 - 8.9\%), respectivamente, además de una correlación inversa con el valor calórico $(\mathrm{r}=-0.8, P<0.05)$.

El valor calórico de $A$. tuberculosa osciló entre 5.02 y $5.37 \mathrm{kcal} / \mathrm{g}$, mientras que para $A$. similis fue entre 5.10 y $5.45 \mathrm{kcal} / \mathrm{g}$.

\section{DISCUSIÓN}

Se determinó que la talla de las poblaciones estudiadas mantuvo una distribución relativamente homogénea durante el periodo de muestreo (Cuadro 1). Aunque la longitud promedio de $A$. similis resultó ser un poco mayor a la de $A$. tuberculosa $(47.9 \pm 2.5 \mathrm{~mm}$ y $46.3 \pm 3.2 \mathrm{~mm}$, respectivamente), no fueron significativamente diferentes $(P<0.05)$, además de encontrarse acorde a la talla estipulada por ley para su extracción (47 mm Decreto Ejecutivo No.
13371-A, de 16 de febrero de 1982) (MAG, 1982). Silva \& Bonilla (2001) en el Golfo Dulce de Costa Rica, al igual que Mora \& Moreno (2008) en la costa ecuatoriana, publicaron longitudes promedio inferiores a las reportadas en este estudio para ambas especies, sin embargo, encontraron la misma tendencia de mayor tamaño de $A$. similis sobre A. tuberculosa.

Los resultados indican que existe una variación significativa entre el porcentaje de los componentes bioquímicos proximales por efecto de la especie (Cuadro 2). Altos porcentajes de humedad fueron encontrados en todas las muestras, hallándose diferencias significativas $(P<0.05)$ entre el valor promedio de $A$. tuberculosa con respecto al registrado en $A$. similis en este trabajo y muy similar con la humedad en otras especies de moluscos bivalvos reportados en el país (Cuadro 2). Estos valores de humedad están considerados como aceptables por ser organismos de vida totalmente acuática (Jiménez-Arce, 
Cuadro 2. Comparación de los valores promedio de los componentes bioquímicos proximales de la carne de Anadara tuberculosa y Anadara similis con Polymesoda radiata, Mytella guyanensis y Tagelus peruvianus

Table 2. Comparison of average values of proximate biochemical components of $A$. tuberculosa and A. similis with Polymesoda radiata, Mytella guyanensis and Tagelus peruvianus

\begin{tabular}{|c|c|c|c|c|c|}
\hline & $\begin{array}{c}\text { Anadara } \\
\text { tuberculosa }^{1}\end{array}$ & $\begin{array}{l}\text { Anadara } \\
\text { similis }^{1}\end{array}$ & $\begin{array}{c}\text { Polymesoda } \\
\text { radiata }^{2}\end{array}$ & $\begin{array}{c}\text { Mytella } \\
\text { guyanensis }^{3}\end{array}$ & $\begin{array}{c}\text { Tagelus } \\
\text { peruvianus }^{4}\end{array}$ \\
\hline Humedad & $83.5^{\mathrm{b}}$ & $78.6^{\mathrm{c}}$ & $90.1^{\mathrm{a}}$ & $80.4^{\mathrm{bc}}$ & $81.6^{\mathrm{bc}}$ \\
\hline Proteínas & $61.6^{\mathrm{b}}$ & $58.6^{\mathrm{b}}$ & $78.3^{\mathrm{a}}$ & $58.6^{\mathrm{b}}$ & $61.7^{\mathrm{b}}$ \\
\hline Lípidos & $8.0^{\mathrm{a}}$ & $9.2^{\mathrm{a}}$ & $9.5^{\mathrm{a}}$ & $7.6^{\mathrm{a}}$ & $8.5^{\mathrm{a}}$ \\
\hline Carbohidratos & $21.6^{\mathrm{a}}$ & $25.6^{\mathrm{a}}$ & $5.78^{c}$ & $22.8^{\mathrm{a}}$ & $15.9^{\mathrm{b}}$ \\
\hline Cenizas & $8.8^{\mathrm{b}}$ & $6.9^{\mathrm{bc}}$ & $6.43^{c}$ & $11.2^{\mathrm{a}}$ & $14.16^{\mathrm{a}}$ \\
\hline Valor calórico & $5.2^{\mathrm{a}}$ & $5.3^{\mathrm{a}}$ & $4.5^{\mathrm{c}}$ & $4.97^{\mathrm{b}}$ & $4.93^{\mathrm{b}}$ \\
\hline
\end{tabular}

a-c Letras diferentes en la misma línea indican diferencias significativas $(P<0.05)$ entre las especies. ${ }^{1}$ Este estudio. ${ }^{2}$ Ruiz et al. (1998); ${ }^{3}$ Cruz y Villalobos (1993); ${ }^{4}$ Fonseca et al (2011).

1993). Porcentajes de humedad semejantes también han sido reportados por Dare \& Edwards (1975) y Fuentes et al. (2009). Con respecto al porcentaje de proteínas en la carne de $A$. tuberculosa y $A$. similis, se presentan diferencias significativas a lo largo del periodo de muestreo, sin embargo, el valor promedio de este componente no fue diferente entre ambas especies, y solo fue superado significativamente por $P$. radiata. El porcentaje de carbohidratos en la carne de $A$. tuberculosa y $A$. similis no mostró diferencias significativas, sin embargo, exhibe un claro patrón estacional que puede deberse a que estos son el principal recurso de energía para la gametogénesis en bivalvos. Diversos estudios respaldan esta hipótesis (Beninger \& Lucas, 1984; Ruiz et al. 1992; Galap et al. 1997; Li et al. 2000; Park et al. 2001; Liu et al. 2008). En nuestra investigación no se consideraron aspectos de reproducción, por lo que es conveniente realizar este tipo de estudios para comprobar lo expuesto. Además, el porcentaje de carbohidratos varía inversamente con los de las proteínas, lo cual concuerda con los resultados obtenidos por Dare \& Edwards
(1975); Bressan \& Marín (1985); PaénzOsuna et al. (1993); Cruz \& Villalobos (1993) y Ruiz et al. (1998).

Los lípidos y las cenizas fueron los componentes minoritarios y no mostraron diferencias significativas entre las especies en estudio. No obstante, fueron semejantes a los porcentajes reportados en la literatura (Cruz \& Villalobos, 1993; Ruiz et al 1998; Ojea et al. 2005; Liu et al. 2008 y Fonseca et al. 2011).

El valor calórico de $A$. tuberculosa y el de $A$. similis fueron de 5.2 y $5.3 \mathrm{kcal} / \mathrm{g}$, respectivamente, valores que se encuentran de acuerdo con otros reportados para invertebrados acuáticos (Winberg, 1971).

Debido a que las especies de bivalvos estudiadas no presentaron diferencias significativas en los porcentajes de proteínas, lípidos, carbohidratos y cenizas, es evidencia suficiente para decir que ambas especies presentan la misma calidad nutritiva. Por otra parte, el escaso contenido de lípidos obtenido para las especies estudiadas en este trabajo hace que estas se consideren una carne magra óptima para el consumo humano. 


\section{AGRADECIMIENTOS}

Esta investigación es una contribución de la Universidad Nacional (Escuela de Ciencias Biológicas), gracias al apoyo financiero del Fideicomiso 32-04 BANCREDITO-IMAS-BANACIO/73-2002 y del Programa de Regionalización Interuniversitaria del Pacífico Central. Se le agradece a Jorge Boza por su colaboración en las correcciones al manuscrito.

\section{BIBLIOGRAFÍA}

Ampie, C. L. \& Cruz, R. A. (1989). Tamaño y madurez sexual de Anadara tuberculosa (Bivalvia: Arcidae) en Costa Rica. Brenesia, 31, 21-24.

AOAC (ASSOCIATION OF OFFICIAL AGRICULTURAL CHEMISTS). (1984). Official methods of analysis of the Association of Official Analytical Chemists. Washington, EE.UU.: Ed. Association of Official Analytical Chemists.

Beninger, P. G. \& Lucas, A. (1984). Seasonal variations in condition, reproductive activity, and gross biochemical composition of two species of adult clam reared in a common habitat: $T a$ pes decussatus L (Jeffreys) and Tapes philippinarum (Adams \& Revé). $J$. Exp. Mar. Biol. Ecol., 79(1), 19-37.

Bressan, M. \& Marín, M. G. (1985). Seasonal variations in biochemical composition and condition index of cultured mussels (Mytilus galloprovincialis Lmk.) in the lagoon of Venice (North Adriatic). Aquaculture, 48(1), 13-21.

Campos, J. A., Fournier, M. L. \& Soto, R. (1990). Estimación de la población de Anadara tuberculosa (Bivalvia: Arcidae) en Sierpe-Térraba, Costa Rica. Rev. Biol. Trop., 38, 477-480.
Carballo-Avendaño, J. E. \& Herrera-Ramírez, C. H. (1996). Composición química, rendimiento y evaluación de la calidad de la piangua (Anadara tuberculosa) almacenada en refrigeración $\left(4^{\circ} \mathrm{C}\right)$. REVITECA, 5, 1-7.

Crisp, D. J. (1971). Energy flow measurements. En N. A. Holme \& A. D. McIntyre (Eds.), Methods for the study of marine benthos (pp. 197-278). Blackwell, Oxford: IBP Handbook.

Cruz, R. A. (1982). Variación mensual del índice de condición del molusco Anadara tuberculosa (Pelecypoda: Arcidae) en Punta Morales, Puntarenas, Costa Rica. Rev. Biol. Trop., 30, 1-4.

Cruz, R. A. \& Palacios, J. A. (1983). Biometría del molusco Anadara tuberculosa (Pelecypoda: Arcidae) en Punta Morales, Puntarenas, Costa Rica. Rev. Biol. Trop., 31, 175-179.

Cruz, R. A. (1984a). Algunos aspectos de la reproducción de Anadara tuberculosa (Pelecypoda: Arcidae) de Punta Morales, Puntarenas, Costa Rica. Rev. Biol. Trop., 32, 45-50.

Cruz, R. A. (1984b). Algunos aspectos reproductivos y variación mensual del índice de condición de Anadara similis (Pelecypoda: Arcidae) de Jicaral, Puntarenas, Costa Rica. Brenesia, 22, 95-105.

Cruz, R. A. \& Villalobos, C. R. (1993). Monthly changes in tissue weight and biochemical composition of the mussel Mytella guyanensis (Bivalvia: Mytillidae) in Costa Rica. Rev. Biol. Trop., 41, 93-96.

Dare, P. J. \& Edwards, D. B. (1975). Seasonal changes in flesh weigth and biochemical composition of mussels (Mytilus edulis) in the Conwy Estuary, North Wales. J. Exp. Mar. Biol. Ecol., 18(2), 89-97. 
Fernández, B. \& Brunker, T. (1977). Estudio bacteriológico de bivalvos del Golfo de Nicoya, Costa Rica. Condición del molusco recién recolectado. Rev. Biol. Trop., 25, 101-107.

Fernández, B. (1983). Estudio bacteriológico de bivalvos del Golfo de Nicoya, Costa Rica II. Condición del molusco al momento de comerlo. Rev. Biol. Trop., 31(2), 311-316.

Fonseca, C., Marín-Vindas, C., Chavarría-Solera, F., Cruz, R. A. \& Toledo, P. (2011). Variación estacional de la composición proximal del mejillón $T a-$ gelus peruvianus (Bivalvia: Solecurtidae) del Golfo de Nicoya, Puntarenas, Costa Rica. Rev. Biol. Trop., 59(4), 1517-1523.

Fuentes, A., Fernández-Segovia, I., Escriche, I. \& Serra, J. A. (2009). Comparision of physico-chemical parameters and composition of mussels (Mytilus galloprovincialis Lmk.) from different Spanish origins. Food Chem., 112(2), 295-302.

Galap, C., Leboulenger, F. \& Grillot, J. P. (1997). Seasonal variations in biochemical constituents during the reproductive cycle of the female dog cockle Glycymeris glycymeris. Mar. Biol., 129(4), 625-634.

García-Domínguez, F. A., De Haro-Hernández, A., García-Cuellar, A., Villalejo-Fuerte, M. \& Rodríguez-Astudi1lo, S. (2008). Ciclo reproductivo de Anadara tuberculosa (Sowerby, 1833) (Arcidae) en Bahía Magdalena, México. Rev. Biol. Mar. Oceanogr., 43(1), 143-152.

Herrero, L., Palacios, F., Alejandro, H. \& Vega, A. F. (1999). Ausencia de detección de enterovirus en bivalvos Anadara tuberculosa (Bivalvia: Arcidae) por contaminación química en el Pacífico de Costa Rica. Rev. Biol. Trop., 47(3), 419-427.

Jiménez-Arce, G. (1993). Composición químico-nutricional en diferentes tallas y sexos del cambute, Strombus gracilior (Megastropoda: Strombidae) de Playa Panamá, Costa Rica. Rev. Biol. Trop., 41(3), 345-349.

Li, Q., Osada, M. \& Mori, K. (2000). Seasonal biochemical variations in Pacific oyster gonadal tissue during sexual maturation. Fish. Sci., 66(3), 502-508.

Liu, W., Li, Q., Yuan, Y. \& Zhang, S. (2008). Seasonal variations in reproductive activity and biochemical composition of the cockle Fulvia mutica (Reeve) from the eastern coast of China. J. Shellfish Res., 27, 405-411.

Lucas, A. \& Beninger, P. (1985). The use of physiological condition indices in marine bivalve aquaculture. Aquaculture, 44, 187-200.

MacKenzie, C. L., Jr. (2001). The Fisheries for Mangrove Cockles, Anadara spp., from Mexico to Peru, with Descriptions of their Habitats and Biology, the Fishermen's Lives, and the Effects of Shrimp Farming. Mar. Fish. Rev., 63(1), 1-39.

Ministerio de Agricultura y Ganadería (MAG). (1982). Talla mínima comercial de la piangua Anadara tuberculosa. (Decreto Ejecutivo $\mathrm{N}^{\circ}$ 13371-A, Gaceta $\left.N^{\circ} 47\right)$. Recuperado en mayo 2012, disponible en www.gaceta.go.cr. Mora, E. \& Moreno, J. (2008). Abundancia y estructura poblacional de Anadara tuberculosa y Anadara similis en las principales áreas de extracción de la costa ecuatoriana. Quito, Ecuador: Instituto Nacional de Pesca.

Ojea, J., Martínez, D., Novoa, S. \& Cerviño-Otero, A. (2005). Ciclo gametogénico de una población de almeja 
japonesa Ruditapes philippinarum (Adams \& Reeve, 1850) en la ría de Camariñas (noroeste de España) y relación con la composición bioquímica mayoritaria. Bol. Inst. Esp. Oceanogr., 21, 337-342.

Páenz-Osuna, F., Zozueta-Padilla, H. \& Osuna-López, J. (1993). Biochemical composition of the oysters Crassostrea iridescens (Hanley) and Crassostrea corteziensis (Hertlein) in the northwest coast of Mexico: Seasonal changes. J. Exp. Mar. Biol. Ecol., 170(1), 1-9.

Park, M. S., Kang, C.-K. \& Lee, P.-Y. (2001). Reproductive cycle and biochemical composition of the ark shell Scapharca broughtonii (Schrenck) in a southern coastal bay of Korea. J. Shellfish Res., 20(1), 177-184.

Rodríguez, E. \& Antillón, F. (1989). Aeromonas spp. y Plesiomonas shigelloides en bivalvos, cieno y aguas del Golfo de Nicoya, Costa Rica. Rev. Biol. Trop., 37(1), 69-73.

Ruiz, C., Abad, M., Sedano, F., GarcíaMartin, L. O. \& López, J. L. (1992). Influence of seasonal environment changes on the gamete production and biochemical composition of Crassostrea gigas (Thunberg) in suspended culture in El Grove, Galicia, Spain. $J$. Exp. Mar. Biol. Ecol., 155(2), 249-262.

Ruiz, E., Cabrera, J., Cruz, R. A. \& Palacios, J. (1998). Composición bioquímica de la carne de Polymesoda radiata (Bivalvia: Corbiculidae) en Costa Rica. Rev. Biol. Trop., 46(3), 646-653.
Silva, B. A. M. \& Bonilla, C. (2001). Abundancia y morfometría de Anadara tuberculosa y A. similis (Mollusca: Bivalvia) en el manglar de Purruja, Golfo Dulce, Costa Rica. Rev. Biol. Trop., 49 (Supl. 2), 315-320.

Skoglund, C. (2001). Panamic province molluscan literature. Additions and changes from 1971 through 2000. I Bivalvia, II Polyplacophora. The Festivus, 32, 1-140.

StatPoint, Inc. (2006). STATGRAPHICS ${ }^{\circledR}$ Centurion XV. Version 15.2.06. Warrenton, EE.UU.: StatPoint, Inc.

Vega, A. J. (1994). Estructura de población, rendimiento y épocas reproductivas de Anadara spp. (Bivalvia, Archidae) en la Reserva Forestal Térraba-Sierpe, Puntarenas, Costa Rica. Con recomendaciones para su manejo. Tesis de Maestría no publicada, Universidad de Costa Rica, San José, Costa Rica.

Villalobos, C. R. \& Báez, A. L. (1983). Tasa de crecimiento y mortalidad en Anadara tuberculosa (Bivalvia: Arcidae) bajo dos sistemas de cultivo en el Pacífico de Costa Rica. Rev. Lat. Acuic., 17, 1-18.

Winberg, G. G. (1971). Methods for the estimation of production of aquatic animals. New York, EE.UU.: Academic Press.

Wong, E., Antillón, F., Glenn, E. \& González, M. I. (1997). Depuración microbiológica artesanal de la piangua, Anadara tuberculosa (Mollusca: Arcidae). Rev. Biol. Trop., 45(4), 1445-1452. 